\title{
Controle de ARroz-Vermelho (Oryza sp.) COM o Herbicida NICOSUlFURON OU A MISTURA FORMULADA DE IMAZETHAPYR + IMAZA PIC ${ }^{1}$
}

\author{
Control of Red Rice (Oryza sp.) with Herbicide Nicosulfuron or Imazethapyr + Imazapic \\ Formulate Mixture
}

\author{
FONTANA, L.C. ${ }^{2}$, AGOSTINETTO, D. ${ }^{3}$, PINTO, J.J.O. ${ }^{4}$, ROSENTHAL, M.D. ${ }^{5}$, RIGOLI, R.P. ${ }^{6}$ e \\ FIGUEREDO, S.S. 2
}

\begin{abstract}
RESUMO - Foi avaliada a eficácia no controle de arroz-vermelho com o herbicida nicosulfuron ou a mistura formulada de imazethapyr + imazapic. O experimento foi conduzido em casa de vegetação da Faculdade de Agronomia Eliseu Maciel (UFPel), no município de Capão do Leão (RS), na estação de crescimento 2005/06, em delineamento experimental de blocos ao acaso, com quatro repetições. Os tratamentos foram arranjados em esquema fatorial $2 \times 7$, sendo o fator $\mathrm{A}$ os herbicidas e o fator $\mathrm{B}$ as doses dos herbicidas $(0,0,25,0,50,0,75,1,0$, 1,25 e 1,50 $\left.\mathrm{L} \mathrm{ha}^{-1}\right)$. A aplicação foi feita sobre as plantas de arroz-vermelho em estádio de desenvolvimento $\mathrm{V}_{4}$. As variáveis analisadas foram controle, estatura da planta e massa seca da parte aérea. Para as variáveis respostas foi verificada interação significativa entre os fatores estudados. Incrementos nas doses dos herbicidas resultaram em respostas crescentes de controle, com redução na taxa de crescimento das plantas e produção de massa seca da parte aérea. Os resultados mostraram que o arroz-vermelho é controlado pelos herbicidas testados. A eficácia de controle não é restrita à mistura formulada de imazethapyr + imazapic, ocorrendo também com o herbicida nicosulfuron a partir de $40 \mathrm{~g} \mathrm{ha}^{-1}$.
\end{abstract}

Palavras-chave: arroz irrigado, inibidor de ALS, imidazolinona, sulfoniluréia.

\begin{abstract}
Red rice control efficacy using nicosulfuron or a formulated mixture of imazethapyr + imazapic was evaluated. A greenhouse experiment was carried out at Faculdade de Agronomia Eliseu Maciel (UFPel) in Capão do Leão(RS), during the 2005/2006 growing season, in a randomized complete block design and four replications. Treatments were arranged in a $2 \times 7$ factorial, with factor $A$ being the herbicides and factor $B$ the herbicide rates $(0,0.25,0.50,0.75,1.0,1.25$ and $\left.1.50 \mathrm{~L} \mathrm{ha}^{-1}\right)$. The herbicides were applied over red rice plants at the $V_{4}$ development stage. Control, plant height and aerial dry mass were evaluated. There was a significant interaction for the variables between the factors studied. Increased herbicide rates resulted in increasing control responses and decreased plant height and dry biomass. Results showed that red rice is efficiently controlled by the herbicides. Control efficacy is not restricted to the formulated mixture of imazethapyr+imazapic, also occurring with nicosulfuron starting from $40 \mathrm{~g} \mathrm{ha}^{-1}$.
\end{abstract}

Keywords: irrigated, rice, ALS-inhibitor herbicide, imidazolinone, sulfonylurea.

Recebido para publicação em 18.4.2007 e na forma revisada em 29.11.2007.

2 Eng-Agr--., Mestranda em Fitossanidade da Universidade Federal de Pelotas - UFPel, Caixa Postal 354, 96010-900 Capão do Leão-RS, <lisicamponogara@gmail.com>. ${ }^{3}$ Engo-Agro․, Dr., Professor da Faculdade de Agronomia Eliseu Maciel da UFPel, bolsista do CNPq. ${ }^{4}$ Eng $^{0}$-Agr ${ }^{\circ}$., M.Sc., Professor da Faculdade de Agronomia Eliseu Maciel da UFPel; ${ }^{5}$ Eng $^{2}$-Agr ${ }^{2}$., Faculdade de Agronomia Eliseu Maciel da UFPel. ${ }^{6}$ Bióloga, Mestranda em Fitossanidade, FAEM/UFPel. 


\section{INTRODUÇÃO}

O arroz-vermelho é considerado uma das mais importantes plantas daninhas em áreas produtoras de arroz no mundo (Eleftherohorinos \& Dhima, 2002). As populações de arroz-vermelho são bastante variáveis com relação às características das plantas e das sementes, sendo utilizadas para diferenciar os biótipos. Assim, os biótipos que apresentam sementes com cariopse de cor palha, marrom ou amarelada são chamados de arroz-vermelho, enquanto os biótipos cujas sementes apresentam cariopse de cor escura ou preta são conhecidos como arroz-preto (Noldin et al., 1999a).

$\mathrm{O}$ arroz-vermelho tem sido tradicionalmente classificado como pertencente à mesma espécie do arroz cultivado, devido à semelhança existente nas características morfológicas. Entretanto, estudos utilizando marcadores de DNA mostraram que essa espécie daninha é, na verdade, constituída de espécies do gênero Oryza, como O. rufipogone $O$. nivara, e subespécies como $O$. sativa ssp. indicae $O$. sativa ssp. japonica (Vaughan et al., 2001).

O arroz-vermelho compete com o arroz cultivado especialmente por luz, podendo também competir por água e nutrientes se estes recursos forem escassos (Agostinetto et al., 2001). Suas características morfológicas, como maior estatura, normalmente conferem a essa planta daninha maior habilidade competitiva (Diarra et al., 1985). A produtividade de cultivares de arroz foi reduzida com o aumento da população de arroz-vermelho, com perda de $755 \mathrm{~kg} \mathrm{ha}^{-1}$ para cada planta $\mathrm{m}^{-2}$ (Ottis et al., 2005). No Rio Grande do Sul (RS), estimativas mostram perdas correspondentes a $20 \%$ da produção de arroz em decorrência da competição com o arrozvermelho (Marchezan et al., 2004); sua mistura com arroz cultivado pode reduzir o preço de comercialização e aumentar os custos de produção, devido ao custo das práticas adicionais de controle adotadas pelo produtor. Além disso, as sementes de arroz-vermelho podem permanecer viáveis no solo por longo período de tempo, tornando difícil sua erradicação (Noldin et al., 2004, 2006).

A similaridade existente entre o arroz-vermelho e o arroz cultivado, até recentemente, impedia o controle seletivo desta planta daninha com herbicidas. O controle químico, embora eficiente, causava grande fitotoxicidade ao arroz cultivado (Noldin et al., 1999b). O manejo de arroz-vermelho era realizado com outros métodos, como uso de sementes de arroz isentas de arroz-vermelho, arranque manual, uso de sementes pré-germinadas, preparo antecipado do solo com semeadura direta e emprego da rotação de culturas (Agostinetto et al., 2001).

Após décadas de busca por opções para controle de arroz-vermelho, desenvolveram-se genótipos de arroz tolerantes a herbicidas do grupo químico das imidazolinonas, inibidores da enzima ALS, o que possibilitou o controle do arroz-vermelho de forma seletiva à cultura (Steele et al., 2002). A variabilidade para esse caráter foi obtida por mutação induzida através da utilização de mutagênico químico (Croughan et al., 1996).

Essa nova tecnologia associa cultivares de arroz tolerantes a herbicidas não-seletivos ao arroz, constituindo-se numa estratégia eficiente para controle de arroz-vermelho (Steele et al., 2002; Ottis et al., 2003; Levy Jr. et al., 2006; Villa et al., 2006), sendo difundida através do sistema denominado Clearfield ${ }^{\circledR}$. No Brasil, esse sistema está sendo utilizado desde a safra 2003/04, com a aplicação do herbicida Only ${ }^{\circledR}$, que é constituído pela mistura formulada de imazethapyr $\left(75 \mathrm{~g} \mathrm{~L}^{-1}\right)$ e imazapic $\left(25 \mathrm{~g} \mathrm{~L}^{-1}\right)$. Na planta, sua absorção é tanto radical como foliar, sendo translocado pelo xilema e floema, acumulando-se nos pontos de crescimento. $\mathrm{O}$ herbicida Only ${ }^{\circledR}$ pertence ao grupo químico das imidazolinonas, tendo como mecanismo de ação a inibição da enzima acetolactato sintase (ALS) ou acetoidróxido sintase (AHAS), primeira enzima da rota de sintese dos aminoácidos de cadeia ramificada, valina, leucina e isoleucina. Essa inibição interrompe a sintese protéica, que, por sua vez, interfere na sintese de DNA e no crescimento celular (Trezzi \& Vidal, 2001). Os sintomas das plantas sob efeito dos herbicidas inibidores da ALS incluem paralisação do crescimento, amarelecimento dos meristemas e redução no crescimento das raízes (Vargas et al., 1999).

No entanto, é de se esperar que o arroz cultivado tolerante ao herbicida imazethapyr possua também tolerância a outros herbicidas 
similares, como imazaquin, imazapyr, nicosulfuron, pyrithiobac e triasulfuron (Webster \& Masson, 2001). Dentre esses, nicosulfuron, imazaquin e imazapyr demonstraram ser seletivos ao arroz mutado e eficientes no controle de plantas daninhas.

O nicosulfuron também pertence à classe dos herbicidas inibidores da ALS, fazendo parte do grupo químico das sulfoniluréias. É um herbicida seletivo utilizado na cultura do milho, de ação sistêmica, aplicado em pós-emergência e recomendado para controlar várias espécies (Andrei, 2005).

Assim, de modo semelhante ao herbicida imazethapyr + imazapic, utilizado no controle de arroz-vermelho, é possivel que outros inibidores da enzima ALS, como o nicosulfuron, também apresentem eficiência no controle dessa planta daninha.

O objetivo deste estudo foi avaliar a eficácia de controle de arroz-vermelho com a utilização do herbicida nicosulfuron.

\section{MATERIAL E MÉTODOS}

O experimento foi realizado em casa de vegetação da Faculdade de Agronomia Eliseu Maciel, da Universidade Federal de Pelotas (UFPel), localizada no município de Capão do Leão, RS, no período de dezembro de 2005 a fevereiro de 2006.

As unidades experimentais constaram de baldes plásticos com capacidade de oito litros, preenchidos com solo peneirado, classificado como Planossolo Hidromórfico Eutrófico Solódico, pertencente à Unidade de Mapeamento Pelotas (Embrapa, 1999). Em cada vaso foram semeadas 25 sementes de arroz-vermelho; após a emergência, procedeu-se ao desbaste, selecionando-se cinco plântulas. O biótipo utilizado apresentava pericarpo de cor avermelhada e ausência de aristas, sendo proveniente de área localizada no município de Pelotas, RS.

O delineamento experimental utilizado foi de blocos casualizados, com quatro repetições. Os tratamentos foram arranjados em esquema fatorial, em que o fator A comparou herbicidas (imazethapyr + imazapic ou nicosulfuron) e o fator B avaliou doses dos herbicidas, correspondentes a 0,00 (testemunha sem aplicação),
$0,25,0,75,1,00,1,25$ e $1,50 \mathrm{~L}^{-1}$ de nicosulfuron $\left(40 \mathrm{~g} \mathrm{~L}^{-1}\right)$ ou imazethapyr + imazapic $(75$ $\left.+25 \mathrm{~g} \mathrm{~L}^{-1}\right)$. Nos tratamentos com o herbicida imazethapyr + imazapic, foi adicionado à calda de pulverização adjuvante não-iônico (Dash $\left.\mathrm{HC}^{\circledR}\right)$ na proporção de $0,3 \% \mathrm{v} / \mathrm{v}$.

A aplicação foi efetuada sobre as plantas de arroz-vermelho em estádio de desenvolvimento $\mathrm{V}_{4}$, identificado pela formação do colar na quarta folha do colmo principal (Counce et al., 2000), com auxílio de pulverizador costal pressurizado a $\mathrm{CO}_{2}$, munido com bico 110.015 do tipo leque, regulado com pressão de $210 \mathrm{kPa}$, que proporcionou a aplicação de $150 \mathrm{~L} \mathrm{ha}^{-1} \mathrm{de}$ calda herbicida.

A irrigação foi realizada de acordo com a necessidade da cultura, sendo mantida lâmina de água permanente de aproximadamente $5 \mathrm{~cm}$ a partir de três dias após a aplicação dos herbicidas.

As variáveis analisadas foram o controle, a estatura de planta e a massa seca da parte aérea. O controle foi observado visualmente aos $7,14,21$ e 28 dias após a aplicação dos tratamentos (DAT), utilizando escala em que zero representou ausência de sintomas e 100 a morte das plantas. A estatura de planta $(\mathrm{cm})$, avaliada aos 14, 21 e 28 DAT, foi obtida pela medida do colmo principal, desde a coroa até a extremidade da última folha com colar formado, com auxílio de régua graduada. Aos 28 DAT, a parte aérea das plantas foi coletada e seca em estufa com circulação forçada de ar a $65^{\circ} \mathrm{C}$, para determinação da massa seca (g).

Os dados obtidos foram avaliados quanto à sua homocedasticidade e, posteriormente, submetidos à análise da variância $(\mathrm{p} \leq 0,05)$; sendo significativa, os dados foram analisados por modelos de regressão linear e não-linear.

\section{RESULTADOS E DISCUSSÃO}

Verificou-se interação significativa entre os fatores estudados para todas as variáveis respostas, exceto para estatura aos 14 DAT, em que a diferença entre os herbicidas não foi significativa.

O controle, em geral, foi superior com o herbicida imazethapyr + imazapic, à exceção das doses mais elevadas, em que o herbicida

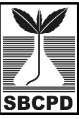

Planta Daninha, Viçosa-MG v. 25, n. 4, p. 783-790, 2007 
nicosulfuron atingiu niveis de controle similares aos obtidos com imazethapyr + imazapic (Figura 1).

O controle de arroz-vermelho ajustou-se à equação linear para o herbicida nicosulfuron e exponencial para imazethapyr + imazapic, com ajuste adequado dos dados aos modelos (Figura 1).

Em todas as avaliações observou-se aumento do controle de arroz-vermelho com incremento na dose dos herbicidas; o herbicida imazethapyr + imazapic proporcionou maior nivel de controle em menores doses, comparativamente ao nicosulfuron (dados das avaliações aos 7,14 e 28 DAT não apresentados). Aos 21 DAT, o valor máximo de controle com imazethapyr + imazapic estimado pelo modelo atingiu 94\%. Já o herbicida nicosulfuron proporcionou controle equivalente ao observado com imazethapyr + imazapic apenas nas maiores doses estudadas, sendo necessária, para controle de arroz-vermelho, a utilização de dose a partir de $1 \mathrm{~L} \mathrm{ha}^{-1}$.

O aumento na eficácia de controle de arroz-vermelho com incremento na dose de imazethapyr aplicado em pós-emergência também foi observado em outros estudos, atingindo con-

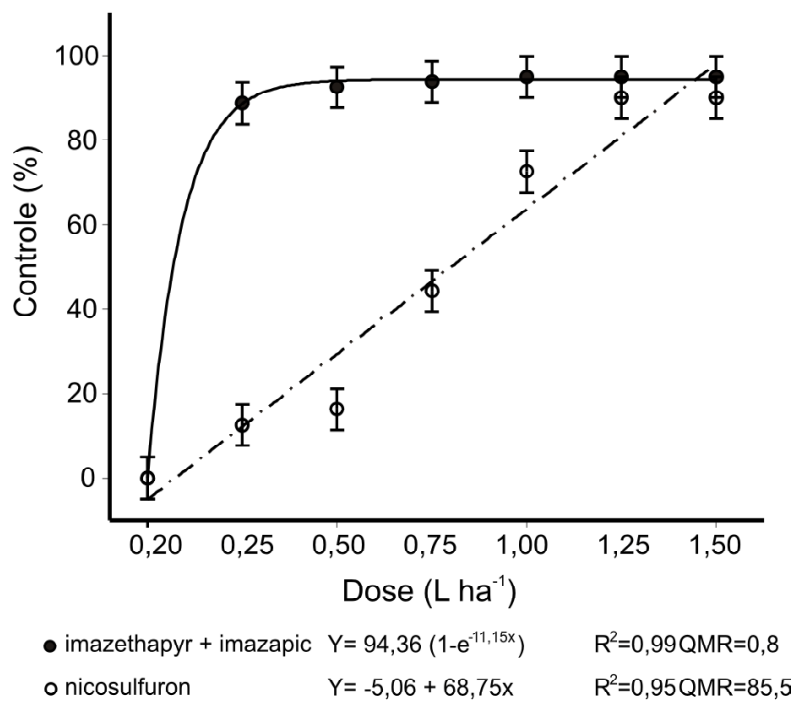

Figura 1 - Controle de arroz-vermelho pelos herbicidas nicosulfuron ou imazethapyr + imazapic, aos 21 dias após aplicação dos tratamentos. FAEM/UFPel, Capão do Leão/ RS, 2005/06. trole superior a $90 \%$ com $70 \mathrm{~g} \mathrm{ha}^{-1}$ (Steele et al., 2002; Ottis et al., 2003).

Durante o período em que as plantas foram avaliadas, observou-se que o controle proporcionado por $1 \mathrm{~L} \mathrm{ha}^{-1}$ evoluiu da primeira para a última avaliação (Figura 2). Isso indica que o arroz-vermelho não mostrou capacidade de se recuperar das injúrias ocasionadas pelo herbicida nicosulfuron ou pela mistura formulada de imazethapyr + imazapic, como observado também em outros estudos (Webster \& Masson, 2001; Steele et al., 2002; Avila et al., 2005).

A eficácia do herbicida imazethapyr no controle de arroz-vermelho já foi reportada em outros trabalhos (Steele et al., 2002; Levy Jr. et al., 2006; Villa et al., 2006). Neste estudo, o herbicida nicosulfuron demonstrou também ser eficiente no controle de arroz-vermelho, corroborando resultados obtidos por Webster \& Masson (2001). No entanto, a eficiência no controle mostrou-se dependente da dose utilizada desses herbicidas.

Para a variável estatura de planta na primeira avaliação, constatou-se semelhança entre os herbicidas em todas as doses testadas (Figura 3). Nas demais avaliações, verificou-

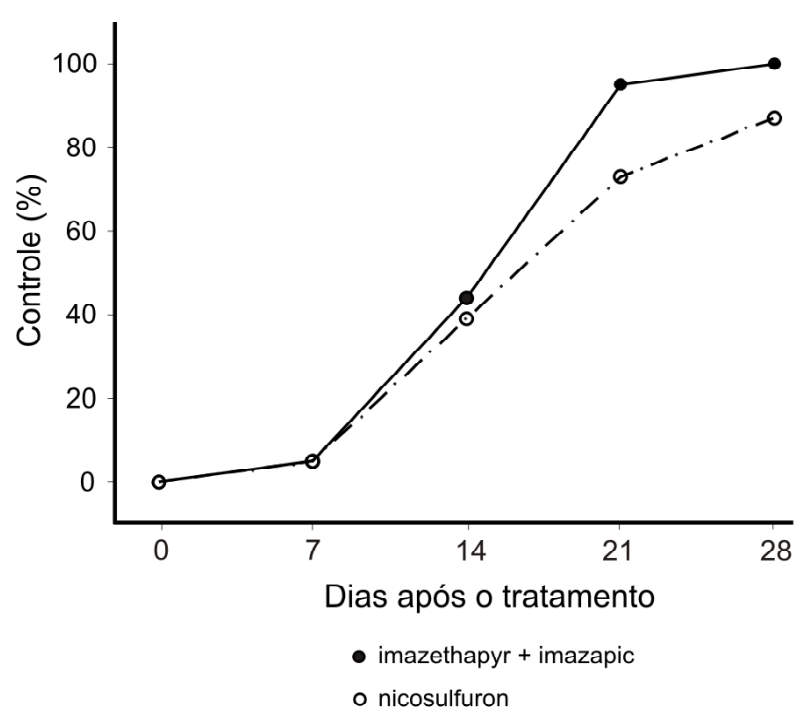

Figura 2 - Evolução no controle de arroz-vermelho pela aplicação dos herbicidas nicosulfuron ou imazethapyr + imazapic na dose de $1 \mathrm{~L} \mathrm{ha}^{-1}$. FAEM/UFPel, Capão do Leão/RS, 2005/06. 
se, em geral, menor estatura pela aplicação de imazethapyr + imazapic comparativamente ao nicosulfuron, à exceção das três doses mais elevadas. Isso se deve ao fato de o arroz-vermelho ter mostrado certa tolerância a doses reduzidas de nicosulfuron.

A variável estatura de planta ajustou-se à equação linear para o herbicida nicosulfuron e exponencial para imazethapyr + imazapic nas avaliações realizadas, exceto para a avaliação aos 14 DAT, em que houve interação, ajustando-se à equação exponencial (Figura 3).

Aos 14 DAT, a redução na taxa de crescimento com o incremento na dose foi semelhante para os dois herbicidas estudados. Nas avaliações seguintes, aos 21 e 28 DAT, o desenvolvimento das plantas permitiu que a diferença entre os efeitos dos herbicidas fosse percebida, observando-se redução na taxa de crescimento com acréscimos à dose do herbicida utilizada. Essa redução foi mais evidente para o herbicida imazethapyr + imazapic, que resultou em menor estatura de arroz-vermelho desde as menores doses estudadas, comparativamente ao nicosulfuron. $\mathrm{O}$ efeito de imazethapyr a partir de doses reduzidas também foi observado em estudos anteriores (Bond et al., 2006; Dal Magro, 2006).

Da mesma forma que para a variável estatura, a massa seca da parte aérea sofreu redução com os tratamentos herbicidas, que foi mais evidente com o herbicida imazethapyr + imazapic, comparativamente ao nicosulfuron, exceto para as duas maiores doses (Figura 4). Em outros estudos também foi observada redução em torno de $90 \%$ na massa seca de arroz-vermelho após aplicação de imazethapyr, imazamox, imazapic, imazapyr, imazaquin, nicosulfuron, primisulfuron e rimsulfuron, demonstrando suscetibilidade das plantas a esses herbicidas (Webster \& Masson, 2001).

Os dados obtidos para a massa seca da parte aérea ajustaram-se à equação linear para o herbicida nicosulfuron e exponencial para imazethapyr + imazapic, com ajuste adequado dos dados aos modelos (Figura 4).

A massa seca foi reduzida com o incremento na dose dos herbicidas estudados; o herbicida imazethapyr + imazapic proporcionou maior redução nas menores doses aplicadas, comparativamente ao nicosulfuron. Isso se deve ao fato de o arroz-vermelho ter mostrado tolerância a pequenas doses de nicosulfuron, conforme discutido anteriormente. A massa seca de cultivares de arroz sem a característica de tolerância às imidazolinonas, IRGA 417, BRIRGA 410, BRS Pelota e Qualimax 1, também foi reduzida exponencialmente com incremento nas doses de imazethapyr + imazapic (Dal Magro et al., 2006).

Os resultados verificados nas avaliações de estatura e massa seca corroboram as observações de controle, permitindo inferir que o arroz-vermelho foi controlado pelos herbicidas imazethapyr + imazapic e nicosulfuron. Portanto, a eficácia de controle não é restrita à mistura formulada de imazethapyr + imazapic, sendo obtida também com a aplicação do herbicida nicosulfuron a partir da dose de $1 \mathrm{~L} \mathrm{ha}^{-1}$ de produto comercial, que corresponde a $40 \mathrm{~g} \mathrm{ha}^{-1}$. 


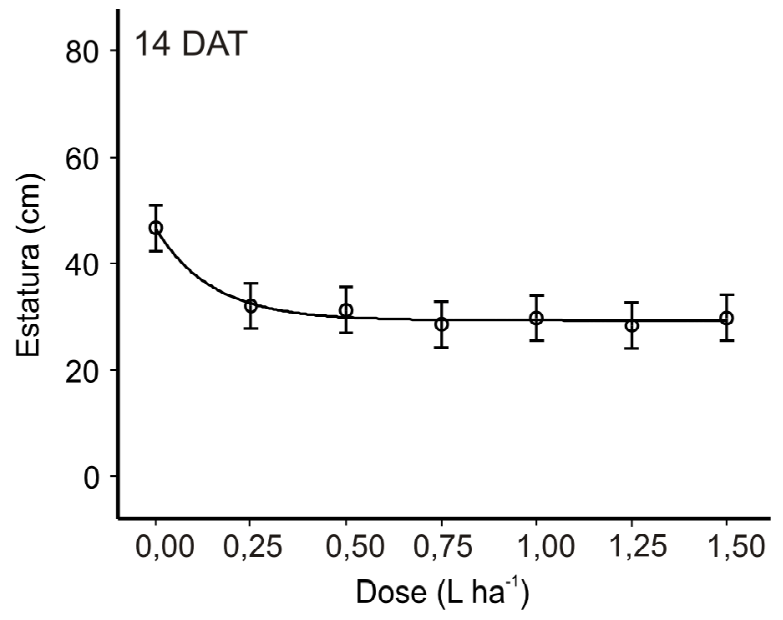

- imazethapyr+imazapic

$Y=29,7+17,6 e^{-4,8 x} \quad R^{2}=0,98 \quad$ QMR $=1,1$

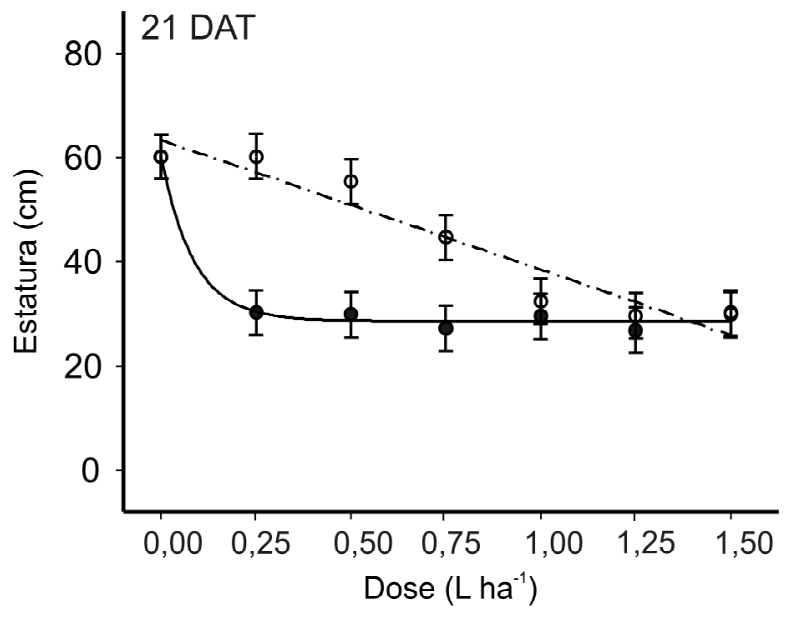

- imazethapyr+imazapic

$Y=28,6+31,5 e^{-11,6 x} \quad R^{2}=0,99 \quad Q M R=19,8$

- nicosulfuron

$Y=63,28-24,8 x \quad R^{2}=0,91 \quad Q M R=20,2$

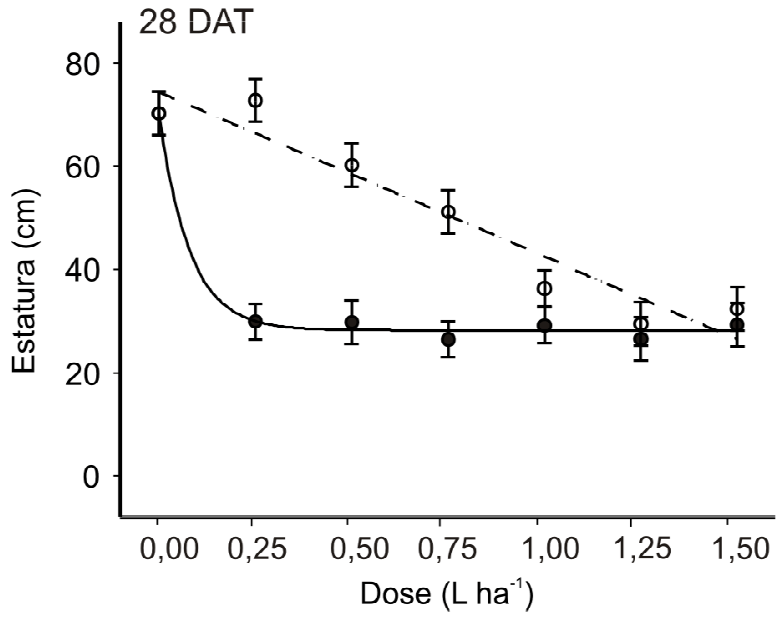

- imazethapyr+imazapic

$Y=28,3+41,9 e^{-12,60 x} \quad R^{2}=0,99 \quad Q M R=10,2$

- nicosulfuron

$Y=74,3-31,9 x \quad R^{2}=0,92 \quad Q M R=30,7$

Figura 3 - Estatura de plantas de arroz-vermelho em função da aplicação de doses dos herbicidas nicosulfuron ou imazethapyr + imazapic. FAEM/UFPel, Capão do Leão/RS, 2005/06. 


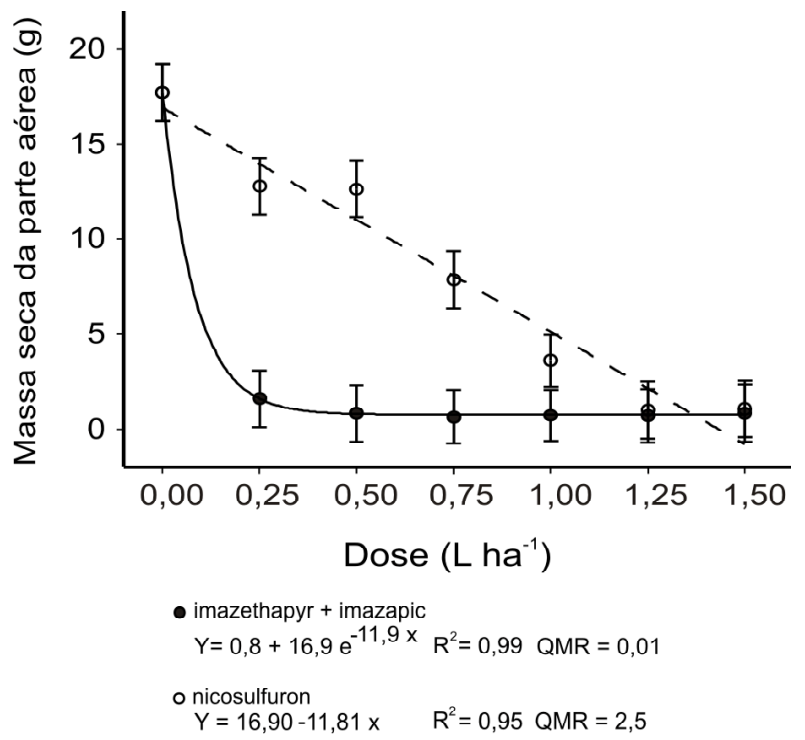

Figura 4 - Massa seca da parte aérea de plantas de arrozvermelho em função da aplicação de doses dos herbicidas imazethapyr + imazapic e nicosulfuron, avaliada aos 28 dias após o tratamento. FAEM/UFPel, Capão do Leão/RS, 2005/06.

\section{LITERATURA CITADA}

AGOSTINETTO, D. et al. Arroz-vermelho: ecofisiologia e estratégias de manejo. Ci. Rural, v. 31, n. 2, p. 341-349, 2001.

ANDREI, E. Compêndio de defensivos agrícolas. 7. ed. São Paulo: Andrei, 2005. 1142 p.

AVILA, L. A. et al. Effect of flood timing on red rice (Oryza spp.) control with imazethapyr applied at different dryseeded rice growth stages. Weed Technol., v. 19, n. 2, p. $479-480,2005$.

BOND, J. A. et al. Corn and rice response to simulated drift of imazethapyr plus imazapyr. Weed Technol., v. 20, n. 1, p. $113-117,2006$.

CROUGHAN, T. P. et al. Herbicide-resistant rice offers potential solution to red rice problem. Louisiana Agric., v. 39, n. 4, p. 10-12, 1996.

COUNCE, P. A.; KEISLING, T. C.; MITCHELL, A. J. A uniform, objective, and adaptative system for expressing rice development. Crop Sci., v. 40, p. 436-443, 2000.

DAL MAGRO, T. et al. Suscetibilidade de cultivares de arroz irrigado (Oryza sativa) à deriva simulada do herbicida imazethapyr + imazapic. Planta Daninha, v. 24, n. 4, p. 751-759, 2006.
DIARRA, A.; SMITH Jr., R. J.; TALBERT, R. E. Growth and morphological characteristics of rice (Oryza sativa) biotypes. Weed Sci., v. 33, n. 3, p. 310-314, 1985.

ELEFTHEROHORINOS, I. L.; DHIMA, K. V. Red rice (Oryza sativa) control in rice (O. sativa) with preemergence and postemergence herbicides. Weed Technol., v. 16, n. 3, p. 537-540, 2002.

\section{EMPRESA BRASILEIRA DE PESQUISA}

AGROPECUÁRIA - EMBRAPA. Centro Nacional de Pesquisa de Solos. Sistema brasileiro de classificação de solos. Rio de Janeiro: 1999. 412 p.

LEVY Jr., R. J. et al. Effect of cultural practices on weed control and crop response in imidazolinone-tolerant rice. Weed Technol., v. 20, p. 249-254, 2006.

MARCHEZAN, E. et al. Controle de arroz-vermelho. In: GOMES, A. S.; MAGALHÃES, A. M. Arroz irrigado no sul do Brasil. Brasília, DF: Embrapa/Informações Tecnológicas, 2004. p. 547-578.

NOLDIN, J. A.; CHANDLER, J. M.; MCCAULEY, G. N. Red rice (Oryza sativa) biology. I. Characterization of red rice ecotypes. Weed Technol., v. 13, n. 1, p. 12-18, 1999a.

NOLDIN, J. A.; CHANDLER, J. M.; MCCAULEY, G. N. Red rice (Oryza sativa) biology. II. Ecotype sensitive to herbicides. Weed Technol., v. 13, n. 1, p. 19-24, 1999 b.

NOLDIN, J. A. et al. Desempenho de populações híbridas $\mathrm{F}_{2}$ de arroz-vermelho (Oryza sativa) com arroz transgênico (O. sativa) resistente ao herbicida amonio-glufosinate.

Planta Daninha, v. 22, n. 3, p. 385-395, 2004.

NOLDIN, J. A.; CHANDLER, J. M.; MCCAULEY, G. N. Seed longevity of red rice ecotypes buried in soil. Planta Daninha, v. 24, n. 4, p. 611-620, 2006.

OTTIS, B. V.; CHANDLER, J. M.; McCAULEY, G. N. Imazethapyr application methods and sequences for imidazolinone-tolerant rice. Weed Technol., v. 17, n. 3, p. 526-533, 2003.

OTTIS, B. V. et al. Rice yield and quality as affected by cultivar and red rice (Oryza sativa) density. Weed Sci., v. 53, n. 4, p. 499-504, 2005.

STEELE, G. L.; CHANDLER, J. M.; McCAULEY, G. N. Control of red rice (Oryza sativa) in imidazolinone-tolerant rice (Oryza sativa). Weed Technol., v. 16, n.3, p. 627-630, 2002.

TREZZI, M. M.; VIDAL, R. A. Herbicidas inibidores da ALS. In: VIDAL, R. A.; TREZZI, M. M. Herbicidologia. Porto Alegre: 2001. p. 25-36. 
VARGAS, L. et al. Resistência de plantas daninhas a herbicidas. Viçosa, MG: Universidade Federal de Viçosa, 1999. $131 \mathrm{p}$.

VAUGHAN, L. K. et al. Is all red rice found in commercial rice really Oryza sativa? Weed Sci., v. 49, p. 468-476, 2001.
VILLA, S. C. C. et al. Controle de arroz-vermelho em dois genótipos de arroz (Oryza sativa) tolerantes a herbicidas do grupo das imidazolinonas. Planta Daninha, v. 24, n. 3, p. $549-555,2006$.

WEBSTER, E. P.; MASSON, J. A. Acetolactate synthaseinhibiting herbicides on imidazolinone-tolerant rice. Weed Sci., v. 49, n. 5, p. 652-657, 2001. 